\title{
Continents manuscrits
}

Génétique des textes littéraires - Afrique, Caraïbe, diaspora

$4 \mid 2015$

La matière Congo

\section{Quand le papier révèle les rapports entre un écrivain et le pouvoir : Nkunga Maniongo de Paul Lomami Tchibamba}

Jean-Pierre Orban

\section{(2) OpenEdition}

Édition électronique

URL : http://journals.openedition.org/coma/519

DOI : 10.4000/coma.519

ISSN : 2275-1742

Éditeur

Institut des textes \& manuscrits modernes (ITEM)

Référence électronique

Jean-Pierre Orban, «Quand le papier révèle les rapports entre un écrivain et le pouvoir : Nkunga Maniongo de Paul Lomami Tchibamba », Continents manuscrits [En ligne], 4 | 2015, mis en ligne le 15 mars 2015, consulté le 16 avril 2021. URL : http://journals.openedition.org/coma/519 ; DOI : https:// doi.org/10.4000/coma.519

Ce document a été généré automatiquement le 16 avril 2021.

Continents manuscrits - Génétique des textes littéraires - Afrique, Caraîbe, dispora est mis à disposition selon les termes de la licence Creative Commons Attribution - Pas d'Utilisation Commerciale - Pas de Modification 4.0 International. 


\title{
Quand le papier révèle les rapports entre un écrivain et le pouvoir : Nkunga Maniongo de Paul Lomami Tchibamba
}

\author{
Jean-Pierre Orban
}

1 On peut disserter sans fin sur l'acte de naissance de la littérature d'imagination congolaise, on ne contestera cependant pas à Paul Lomami Tchibamba le titre d'un des pères de cette littérature, et cela, de part et d'autre du fleuve Congo. Car, de sa naissance à Brazzaville en 1914 d'un père lulua du Kasaï (en actuelle RDC) ayant fui la colonie belge à sa mort à Kinshasa en 1985, sa vie fut ponctuée d'allers-retours entre les deux Congos. En 1921, il suit son père à Léopoldville. Après une enfance dans la rue, des études au petit Séminaire, une carrière de dactylographe et des collaborations à des revues pour " évolués », il retraverse le fleuve en 1949 et, peu après, est nommé, sous la tutelle de l'Administration française, à la tête de la revue Liaison, où des auteurs tels que Tchicaya U Tam'si, Jean-Baptiste Tati-Loutard ou Sylvain Bemba feront quelquesuns de leurs premiers pas. En 1961, après les indépendances des deux Congos, il rejoindra Léopoldville, non sans voir ses espoirs de s'y épanouir déçus.

2 En 1948, il participe à un concours littéraire organisé, dans le cadre de la "Foire coloniale de Bruxelles ", par des gens de lettres et un éditeur belge à destination des «indigènes » du Congo belge et du Ruanda-Urundi. Il le remporte avec Ngando, que certains ${ }^{1}$ appellent roman, d'autres, tel Mukala Kadina-Nzuji ${ }^{2}$, récit, mais que l'auteur lui-même nomme conte dans son "Avertissement au lecteur $»^{3}$ qu'il entame par ces mots : «Le contenu des pages qui vont suivre n'est qu'un modeste et tâtonnant essai d'un travail d'imagination.» Histoire qui entrelace le réel et le merveilleux, Ngando constitue un des textes fondateurs de la littérature congolaise, ainsi que le note Alain Mabanckou dans sa Préface à Ah!Mbongo ${ }^{4}$ du même Lomami Tchibamba :

Dans les années quatre-vingt, la lecture de Ngando me bouleversa, d'autant que je découvrais en ce temps-là les grands textes latino-américains portés par le « réalisme merveilleux ». Et je ne cessais de me demander : qu'est-ce que Ngando, 
sinon une sorte de Contes d'amour, de folie et de mort d'Horacio Quiroga ou alors Pedro Páramo de Juan Rulfo? De même que ce dernier auteur allait inspirer toute une génération d'auteurs latino-américains - y compris l'auteur de Cent ans de solitude, Gabriel García Marquez -, Lomami Tchibamba aura plus qu'un impact sur les auteurs congolais des deux rives.

Dans les années cinquante, Paul Lomami Tchibamba entame la rédaction de plusieurs textes dont deux paraîtront plus tard: Ah! Mbongo et Londema ${ }^{5}$. Les débuts de leur écriture datent au moins de 1957 et selon toute vraisemblance antérieurement, car un numéro spécial de la revue Présence Africaine y fait allusion, qui est publié à la suite du Premier Congrès, à Paris, des Écrivains et artistes noirs de 1956 auquel Paul Lomami Tchibamba participe. Euvre ample qui a toutes les caractéristiques du roman, Ah! Mbongo sera finalement publié à titre posthume en 2007, après bien des mésaventures éditoriales. Il constitue, dans le genre romanesque, l'entreprise la plus ambitieuse connue et entamée dans les années cinquante, pour ce qui est du Congo-Kinshasa à tout le moins.

4 Revenu sur la "terre de ses ancêtres ${ }^{6}$ », Paul Lomami Tchibamba observe la dérive du Congo-Léopoldville après l'indépendance et suit les tentatives sécessionnistes ainsi que les rébellions d'inspiration marxiste qui embraseront, en 1963, le Sud-Est du pays avec le leader Pierre Mulele ${ }^{7}$, puis l'Est et le Nord-Est lors de mouvements eux-mêmes inspirés par la révolte «muleliste » sous la direction, entre autres, de Gaston Soumialot et Nicolas Olenga, où les rebelles s'identifieront sous le nom de «simbas » (lions). Le point culminant - en même temps que la fin - de cette dernière rébellion constituera la conquête de la ville de Stanleyville (aujourd'hui Kisangani) et la prise en otage de sa population du 5 août au 24 novembre 1964, avant que la cité soit reprise par le pouvoir central appuyé par une opération aéroportée belgo-américaine 8 .

Ces mouvements de rébellion, et en particulier celui des «simbas» dans le Nord-Est, plus précisément encore dans la province de l'Uele (car il n'est pas fait mention de la prise de Stanleyville) inspireront à Paul Lomami Tchibamba le seul texte n'appartenant ni à sa veine de "réalisme merveilleux" (ainsi Ngando), ni à celle, socio-historique (ainsi Ah!Mbongo) qui exprime le regret par l'auteur de la perte des valeurs ancestrales de l'Afrique tombée dans une course à la modernité induite par l'arrivée du colonisateur. Pas non plus ou peu de dénonciation de l'oppression coloniale comme dans nombre de ses textes. Dans Nkunga Maniongo', Paul Lomami Tchibamba prend à bras-le-corps une réalité politique contemporaine, pour la première fois post-coloniale, fondée sur des faits historiques précis pour dénoncer, certes sur le ton ironique qui est le sien également, le dévoiement de l'Indépendance à laquelle a accédé le Congo en 1960. «PLT écrit, à l'instar d'Amadou Kourouma [...], l'anti-épopée des indépendances africaines ", commente Antoine Tshitungu Kongolo dans sa présentation de La Saga des Bakoyo Ngombé et autres inédits, qui comprend Nkunga Maniongo. "Pour user d'une formule simpliste mais tentante : Nkunga Maniongo, c'est la dénonciation des "soleils des indépendances" sous les tropiques congolaises ${ }^{10}$. »

Pour que son texte soit tout à fait la dénonciation des « soleils des indépendances » à la mode kouroumienne, Paul Lomami Tchibamba a cependant, selon toute vraisemblance, dû en réécrire la fin pour ne pas laisser croire à une quelconque complaisance à l'égard des maîtres du nouveau régime qui, sous la houlette du nouveau président Mobutu, sombrèrent dans les mêmes travers que leurs prédécesseurs. 
7 On ne connaît pas la date exacte de l'écriture de Nkunga Maniongo. Les proches de l'auteur n'avancent aucune hypothèse: Paul Lomami Tchibamba était un écrivain secret, se cachant même de ses proches. Deux éléments, cependant, dans le texte permettent de donner une date minimum. Dans une note ${ }^{11}$, l'auteur qui, régulièrement, mêle à sa fiction des annotations objectives, fait référence à un mémoire universitaire présenté en juillet 1968 à l'université de Lovanium. Élément ultérieur et plus général, l'auteur applique, pour les noms de lieux, les dispositions édictées, dans le cadre d'un «recours à l'authenticité », par le président Mobutu le 27 octobre 1971. Ainsi, il écrit «Baya nDudi avait été envoyé à Kisangani (à l'époque, Stanleyville) en $1953^{12}$ » et, deux paragraphes suivants, «une route partant d'Isiro (ci-devant Paulis)» et « une autre venant de Mahagiport (du ci-devant Lac Albert)», "ci-devant» devant être compris comme « à l'époque ». On sait, par son entourage ${ }^{13}$, et la consultation de ses $\operatorname{archives}^{14}$, que Paul Lomami Tchibamba, suivant les habitudes du dactylographe qu'il avait été, écrivait directement ses œuvres à la machine à écrire, les réécrivait rarement et les corrigeait ensuite, quand il le faisait, à la main. On peut donc considérer le tapuscrit original de ses archives comme la version première et définitive à la fois de Nkunga Maniongo. À une exception près, cependant, dont témoignent les trois dernières pages.

Ces trois pages finales d'un tapuscrit, pour le reste pratiquement intact, sont dactylographiées sur des papiers différents.

Le tapuscrit de Nkunga Maniongo, feuillet 62.

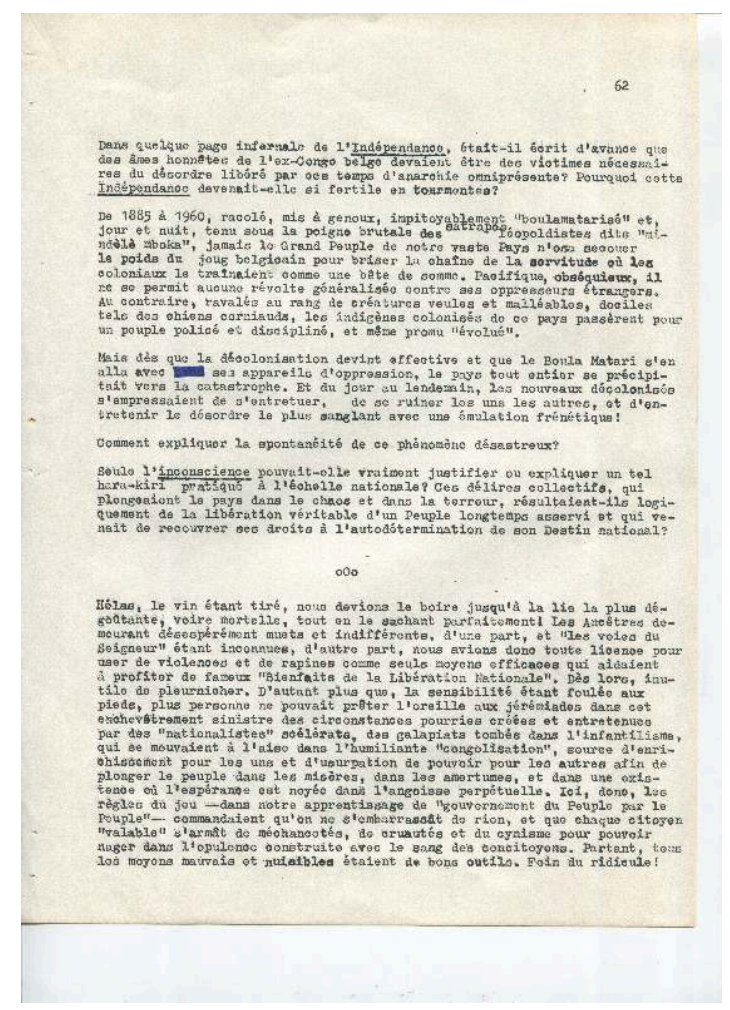




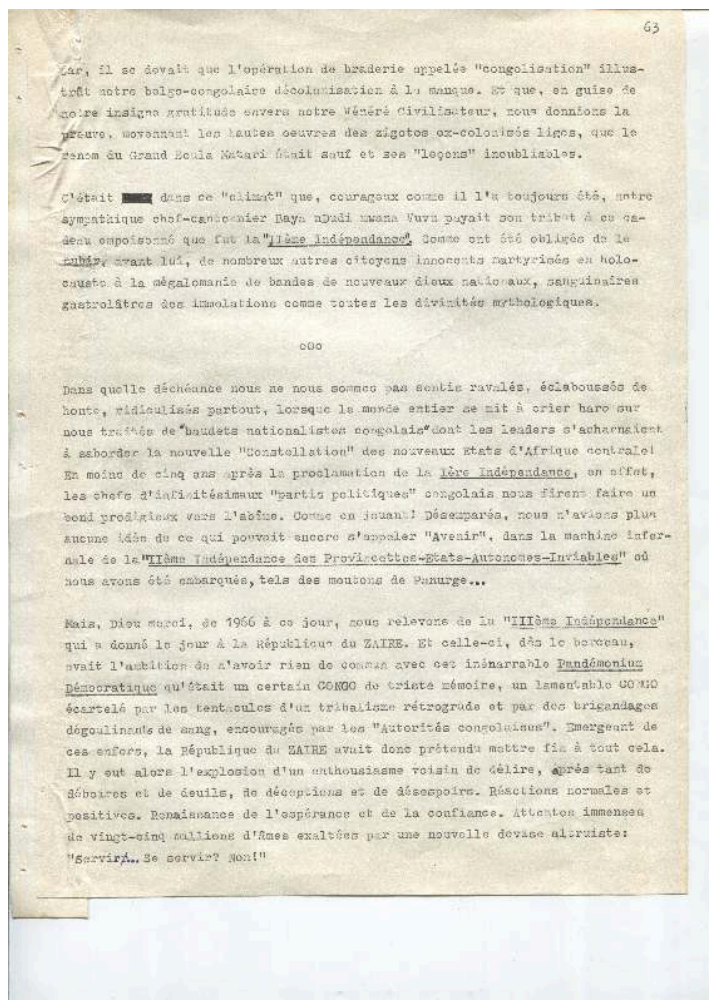

Page dactylographiée sur un feuillet moins large et légèrement moins haut d'un papier plus fin que le reste du document (ainsi la page 62 ci-dessus). La frappe est également différente, avec un interligne plus grand.

9 Le feuillet (et la page) 63 est collé sur une bande qui permet de le convertir au format du reste du tapuscrit et donc de lui être agrafé, ainsi qu'en témoignent les marques des agrafes. Ce feuillet est manifestement, par la trace de l'encre et la finesse de la frappe, l'original. Les deux feuillets suivants, 64 et 65 , constituent, en toute apparence, les copies sur papier pelure de l'exemplaire premier. Ils présentent la même frappe et le même interligne que le feuillet 63.

Que révèle ce changement de papier et d'interligne? L'hypothèse, étayée par le contenu, que ces feuillets sont ultérieurs au tapuscrit original et ont remplacé, à un moment indéterminé, les feuillets originaux.

Que dit le texte? Le début du feuillet (et page) 63 conclut la fin des mésaventures du protagoniste du roman, Baya nDudi, mésaventures narrées dans une longue analepse qui a occupé l'essentiel du texte. Des mésaventures qui se déroulent dans le cadre historique des années 1960-1966, soit depuis l'indépendance du Congo ex-belge. Un contexte que l'auteur évoque sous le terme de "congolisation ", au cours ou à la suite de laquelle eut lieu la tentative, menée par les mouvements rebelles, d'offrir au pays une "II ${ }^{\text {ème }}$ Indépendance ", censée être cette fois réelle, débarrassée du joug des puissances occidentales qui se serait poursuivi après la "première " indépendance: "C'était dans ce "climat" que, courageux comme il l'a toujours été ", écrit Paul Lomami Tchibamba après avoir embrayé sur le terme de "congolisation» utilisé à la page précédente, elle intacte :

[...] notre sympathique chef-cantonnier Baya nDudi mwana Vuvu paya[it $\left.{ }^{15}\right]$ son

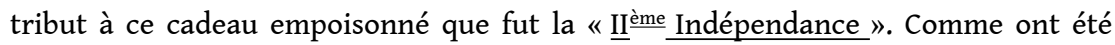
obligés de le subir, avant lui, de nombreux autres citoyens innocents martyrisés en 
holocauste à la mégalomanie de bandes de nouveaux dieux nationaux, sanguinaires gastrolâtres des immolations comme toutes les divinités mythologiques.

Et, après un espace marqué du signe « o0o », il poursuit :

Dans quelle déchéance nous ne nous sommes pas sentis ravalés, éclaboussés de honte, ridiculisés partout [...]. En moins de cinq ans après la proclamation de la I è̀re Indépendance, en effet, les chefs d'infinitésimaux «partis politiques" congolais nous firent faire un bond prodigieux vers l'abîme. Comme en jouant ! Désemparés, nous n'avions plus aucune idée de ce qui pouvait encore s'appeler " Avenir », dans la machine infernale de la " II ${ }^{\text {ème }}$ Indépendance des Provincettes-Etats-AutonomesInviables » où nous avions été embarqués, tels des moutons de Panurge...

L'auteur poursuit ensuite par la période qui a suivi cette tentative de "deuxième indépendance" et les troubles qu'elle a occasionnés. Il le fait d'abord par un affirmation qui se révèle positive : "Mais, Dieu merci, de 1966 [année qui suit la prise de pouvoir de Joseph Désiré Mobutu et est marquée par la neutralisation de tous les contre-pouvoirs et la pendaison d'opposants à la Pentecôte] à ce jour, nous relevons de la "III ème Indépendance" qui a donné le jour à la république du ZAIRE. » La phrase est au présent. La suite est écrite au passé, comme si l'auteur dressait, ultérieurement, un inventaire de cette république et «III ${ }^{\text {ème }}$ Indépendance » dont il voulait se distancier :

Et celle-ci, dès le berceau, avait l'ambition de n'avoir rien de commun avec cet inénarrable Pandémonium Démocratique qu'était un certain CONGO de triste mémoire [...]. Emergeant de ces enfers, la République du ZAIRE avait donc prétendu mettre fin à tout cela.

Suit alors un réquisitoire que rien avant n'annonçait. :

[...] les grands profiteurs du NOUVEAU REGIME [...] s'empressaient d'emporter fébrilement et d'aller trimbaler à travers les villes de l'ex-métropole coloniale des malles et des valises bourrées de gros billets de banque", aux quatre coins cardinaux du ZAIRE fusaient des gémissements de désespoir chez des millions de «Concitoyens-à-part-entière » livrés au déferlement du terrorisme mis en branle, de part et d'autre, par des «nationalistes libérateurs " contre des «nationalistes dominateurs " tous s'acharnant, avec zèle, à massacrer des citoyens sans défense qui, par ailleurs, souffraient de la torture de la faim, enlisés dans un chômage sans issue, ne sachant plus à quel autre «NOUVEAU REGIME » se fier pour trouver la sécurité et pour avoir de quoi manger. [...] Ainsi, hélas, après les tragiques convulsions de notre honteuse congolisation, nous nous retrouvions à nouveau de plain-pied dans la morbidité d'une certaine zaïrisation paupérisante [...]. Ainsi, d'un[e] NOUVEAU REGIME à l'autre, nos gouvernants n'ont pas du tout changé leurs mœurs pillardes [...] condamnant par habitude la communauté nationale à tous les degrés de la misère pendant qu'ils roulaient carrosse et naviguaient dans l'opulence en témoignage éloquent «d'immenses richesses» du ZAIRE, leur propriété exclusive à eux « Honorables Hommes d'Etat » marionnettes entièrement à la merci de prestidigitateurs internationaux.

Et l'auteur de conclure le "nkunga maniongo » (la complainte) de l' « infortuné Baya nDudi mwana Vuvu » par ce cri : "Qui sauvera notre cher pays de la ruine fatale? »

Dans tout le récit qui a précédé et décrit les ravages de la tentative, dévoyée à ses yeux, de « deuxième indépendance », Paul Lomami Tchibamba a, en ce sens, adopté la même position que ceux qui allaient mettre fin à ces " délires collectifs ${ }^{16}$ ", à savoir le général Mobutu et ses soutiens. On peut penser que le récit se terminait par le retour à l'ordre, ou simplement par la fin tragique du héros que l'on retrouve pendu. Or, les trois dernières pages opèrent comme un début de nouveau récit qui, en accéléré, constitue une critique virulente du régime mobutiste. 

régime (qui rétablit temporairement l'économie et la sécurité), ces feuillets d'une autre texture dactylographiés d'une nouvelle manière offrent une critique qui ne pouvait être menée qu'après des années de pratique du nouveau régime zaïrois. Tout laisse penser que les deux parties ont été écrites séparément, en des temps différents. montre sa phrase «Mais, Dieu merci, de 1966 à ce jour, nous relevons de la "III ème Indépendance" qui a donné le jour à la république du ZAIRE », approuver le retour à l'ordre du nouveau régime mobutiste, il ne s'est pas privé de critiquer avec sévérité ce même régime par la suite, dans les nouvelles pages. Et ce, alors que le régime était encore tout-puissant, puisque Paul Lomami Tchibamba décède en 1985 et que le régime du président Mobutu tombe seulement douze ans plus tard, en 1997. Même si le texte ne fut pas publié de son vivant, par le seul fait de l'écrire, Paul Lomami Tchibamba prenait donc le risque encouru par ceux qui, dans le «"nouvel empire du silence", pos[aient] des questions équivalant "à une grave subversion politique" ", comme il le décrit au feuillet 64 de son tapuscrit ${ }^{17}$ : "un plus ou moins long séjour dans les oubliettes de quelque ergastule où des spécialistes s'empressaient de SERVIR des torgnoles aux malaisés. »

Ceci conforte le témoignage de ses proches. En 1984, Paul Lomami Tchibamba était malade et avait sombré dans la pauvreté. L'association des écrivains du Zaïre parvint à lui obtenir une rencontre avec le président Mobutu. Celui-ci reçut, en compagnie d'un de ses fils, celui qu'il connaissait en tant que journaliste et écrivain (en 1982, le président Mobutu avait financé la coédition, par les Editions Lokolé, du recueil reprenant Ngando chez Présence Africaine). Il l'aurait alors absous de ses critiques en raison de son âge et de son état de santé, bien qu'il eût eu, aurait-il dit, toutes les raisons de le jeter en prison. À son tour, Paul Lomami se fit pardonner les critiques qu'il avait écrites ou dites sur lui. Mobutu lui avait alors payé le voyage vers la Belgique pour s'y faire soigner. Paul Lomami s'y était rendu avant de mourir en août 1985.

En Afrique post-coloniale, les relations entre les écrivains et le pouvoir furent et sont complexes, faites de disgrâces et de retours en grâce. L'histoire supposée du tapuscrit de Nkunga Maniongo en témoigne à sa façon.

\section{NOTES}

1. Ainsi Silvia Riva dans Nouvelle histoire de la littérature du Congo-Kinshasa, version française fondée sur une traduction de l'italien par Collin Fort, Paris, L'Harmattan, collection « L'Afrique au cœur des lettres ", 2006, notamment p. 35 et 85.

2. Dans sa Préface à Lomami Tchibamba, Ngando et autres récits, Paris-Kinshasa, Présence Africaine, Éditions Lokolé, 1982, p. 7-11.

3. Lomami Tchibamba, Ngando et autres récits, op. cit., p. 15-20.

4. Paul Lomami Ah! Mbongo, Ah! L'Argent, Paris, L'Harmattan, Collection «L'Afrique au cœur des lettres ", 2007, p. 5.

Continents manuscrits, 4 | 2015 
5. Londema paraîtra une première fois en 1974 sous ce titre dans la revue Cultures au Zaïre et en Afrique, Kinshasa, ONRD, ensuite sous celui de Légende de Londema, suzeraine de Mitsoué-ba-Ngomi dans la même revue en 1975, enfin sera réédité sous ce dernier titre dans Ngando et autres récits, op. cit.

6. Eliane Tchibamba, Une vie au creux des mots : Paul Lomami Tchibamba vu par sa fille, in Ah! Mbongo, Ah! L'Argent, op. cit., p. 11.

7. Voir Benoît Verhaegen, Rébellions au Congo, Bruxelles, CRISP, Kinshasa, IRES, vol. 1, 1966, vol. 2, 1969.

8. Voir notamment Frans Quinteyn, Stanleyville sous la terreur Simba, Mateka, Le Temps des ombres, avant-propos de Benoît Verhaegen, préface de Jean-Pierre Orban, Paris, L'Harmattan, Collection «Congo-Zaïre - Histoire et Société », 2004.

9. « La complainte».

10. La Saga des Bakoyo Ngombé et autres inédits, Paris, L'Harmattan, collection «L'Afrique au cœur des lettres", 2014, p. 11.

11. Idem, p. 170.

12. Idem, p. 117.

13. Sa veuve, Madame Lukuma Mosa'Olongo, et sa fille Eliane Tchibamba.

14. Déposées en 2014 aux Archives et Musée de la littérature, au sein de la Bibliothèque Royale Albert $\mathrm{I}^{\mathrm{er}}$ à Bruxelles. Voir la contribution de Jean-Claude Kangomba, rubrique «Répertoire de fonds d'archives ", url : http://coma.revues.org/514.

15. Correction effectuée lors de l'établissement du texte pour l'édition.

16. La Saga des Bakoyo Ngombé, op. cit., p. 192.

17. Idem, p. 195.

\section{AUTEUR}

\section{JEAN-PIERRE ORBAN}

Ecrivain (Vera, Mercure de France, 2014), dirige la collection « pulsations » aux éditions Vents d'Ailleurs 